GANIT J. Bangladesh Math. Soc. (ISSN 1606-3694) 39 (2019) 87-100

DOI: https://doi.org/10.3329/ganit.v39i0.44160

\title{
ANALYTICAL SOLUTION OF LIÉNARD DIFFERENTIAL EQUATION USING HOMOTOPY PERTURBATION METHOD
}

${ }^{1}$ Md. Mamun-Ur-Rashid Khan and ${ }^{2}$ Goutam Saha

Department of Mathematics, University of Dhaka, Dhaka-1000, Bangladesh

Email: ${ }^{1}$ mamun.math@du.ac.bd, ${ }^{2}$ gsahamath@du.ac.bd

Received: 22-07-2018

Accepted: 24-03-2019

\begin{abstract}
In this research work, the well-known Homotopy perturbation method (HPM) is used to find the approximate solutions of the nonlinear Liénard differential equation (LDE) using different types of boundary conditions. In order to find the accuracy of the approximate solution, one term, two terms and three terms HPM approximations are considered. This idea is actually based on the idea of Taylor's series polynomials. It is found that solution converges to the actual solution with the increase of the terms in the guess solution. Moreover, in each of the new HPM solution, previously obtained solutions are added to it in order to find the exactness of HPM solutions. However, the nature of the solution seems to be complicated. In addition, comparisons are made with the previously published results and a good agreement is observed.
\end{abstract}

Keywords: Homotopy Perturbation method (HPM); Liénard differential equation; Nonlinear differential equations; Analytic solution; Initial and boundary conditions.

\section{Introduction}

The general Liénard differential equation $[1,2]$ can be written as

$$
y^{\prime \prime}+s(x) y^{\prime}+t(x)=h(x) .
$$

which is considered as a generalization of the damped pendulum equation or a damped springmass system [1]. One can use different arbitrary functions for $s(x), t(x)$ and $h(x)$. Such combination refers to different nonlinear models which can be used in many practical applications. It is to be noted that particular choices of $s(x)=\mu\left(x^{2}-1\right), t(x)=x$, and $h(x)=0$ leads to Van der Pole quation treated as a nonlinear model of electronic oscillation [1]. In this work, the special case of LDE (studied by Kong [3]) is solved using HPM with different types of initial and boundary conditions. And Liénard equation is presented below:

$$
y^{\prime \prime}(x)+p y(x)+q y^{3}(x)+r y^{5}(x)=0 .
$$

where $p, q, r$ are real constants.

(C) GANIT: Journal of Bangladesh Mathematical Society, 2019 
The concept of HPM was first established by He [4, 5], which has been used to solve a large number of non-linear problems. It is found that such approximations rapidly converge to the exact solution. Since it is not easy to find the solution of equation (1.2) by usual methods such as the perturbation method, separation of variables etc., HPM can be used to find the analytical solution of nonlinear differential equations with different types of initial and boundary conditions [6-9]. 'In addition, the merging of perturbation method and the Homotopy method is called HPM, which has banished the deficiency of the traditional perturbation methods. In the other hand, this method can take the full benefit of perturbation techniques' $[4,5]$.

Researchers are trying to find the exact solution of Liénard differential equation over some decades and several approximate solutions are proposed. Some of the literatures are presented hereafter.

In 1982, Villari [10] proposed a new criterion for the existence of periodic solution of Liénard Differential Equation. In the next year, he presented a new theorem which showed the assurance of the existence of such solution and few years later, he also presented the qualitative behavior of periodic solution of Liénard Differential Equation [11, 12]. In 1995, Kong [3] studied a particular form of LDE as shown in Eq. (1.2). He showed that explicit solution of this type of equation can be obtained when $p<0, r \geq 0$ and $(q, r) \neq(0,0)$. In 2002 and 2004, Feng [2, 13] worked on the extension of the Kong's [3] work and provided more explicit solutions for different cases. In 2008 and 2012, Matinfar et al.[1, 14] solved the initial value problem of LDE proposed by Kong [3] using variational iteration and differential transform method respectively. They proposed new approximate solutions of equation (1.2).

From the above literature reviews, it is clear that solution of particular type of different boundary value problems of LDE is not investigated for real parameters $p, q$ and $r$. However, exact solution of such types is still an issue to the mathematicians. Therefore, the goal of the present research is to find the approximate exact solution of LDE with different combinations of Neumann and Dirichlet type boundary conditions using HPM. Moreover, such findings will be helpful in the applications of LDE.

\section{Validation Works}

In this section, the special type of Liénard equation is solved by HPM and comparisons between the HPM solution and exact solutions are presented hereafter.

Example 1:

$$
\begin{aligned}
& y^{\prime \prime}(x)+p y(x)+q y^{3}(x)+r y^{5}(x)=0 . \\
& y(0)=\sqrt{-\frac{2 p}{q}}, \quad y^{\prime}(0)=-\frac{p \sqrt{-p}}{\sqrt{-\frac{2 p}{q}}}
\end{aligned}
$$


Let us consider, $r=-3, p=-1, q=4$, then the exact solution [1] is

$$
y(x)=\sqrt{\frac{-2 p(1+\tanh (\sqrt{-p} x))}{q}} .
$$

By using HPM, we have

$$
y^{\prime \prime}-y-\left(y_{0}^{\prime \prime}-y_{0}\right)+t\left(y_{0}^{\prime \prime}-y_{0}\right)+t\left[4 y^{3}-3 y^{5}\right]=0 .
$$

Putting,

$$
y=y_{0}+t y_{1}+t^{2} y_{2} \text { with } y_{0}=\frac{2+x}{2 \sqrt{2}} .
$$

InEq. (3.1) and equating the coefficients of $t$ from both sides, we get

$$
\begin{gathered}
y_{0}^{\prime \prime}-y_{0}+\frac{2+x}{2 \sqrt{2}}=0 \quad \text { with } y_{0}(0)=\frac{1}{\sqrt{2}}, y_{0}^{\prime}(0)=\frac{1}{2 \sqrt{2}} . \\
y_{1}^{\prime \prime}-y_{1}-\frac{2+x}{2 \sqrt{2}}+4 y_{0}^{3}-3 y_{0}^{5}=0 \quad \text { with }_{1}(0)=0, y_{1}^{\prime}(0)=0 . \\
y_{2}^{\prime \prime}-y_{2}+12 y_{0}^{3} y_{1}-15 y_{0}^{5} y_{1}=0 \quad \text { with } y_{2}(0)=0, y_{2}^{\prime}(0)=0 .
\end{gathered}
$$

Solving we get,

$$
\begin{gathered}
y_{0}=\frac{2+x}{2 \sqrt{2}} . \\
y_{1}=\frac{e^{-x}\left(-12+796 e^{2 x}-e^{x}\left(784+808 x+408 x^{2}+148 x^{3}+30 x^{4}+3 x^{5}\right)\right)}{128 \sqrt{2}} .
\end{gathered}
$$

$y_{2}=\frac{1}{65536} e^{-x}\left(3\left(-7607658+2829 x+3981 x^{2}+2590 x^{3}+879 x^{4}+150 x^{5}+10 x^{6}\right)+\right.$ $e^{2 x}\left(-1246283970-166563 x-62685 x^{2}+54526 x^{3}+55521 x^{4}+17910 x^{5}+1990 x^{6}\right)+$ $6 e^{x}\left(211517824+203936512 x+105796544 x^{2}+34000256 x^{3}+8804976 x^{4}+\right.$ $\left.\left.1685408 x^{5}+284856 x^{6}+36704 x^{7}+4094 x^{8}+300 x^{9}+15 x^{10}\right)\right)$.

Thus the two term solution by HPM is $Y=y_{0}+y_{1}$ and the three term solution by HPM is $Y=$ $y_{0}+y_{1}+y_{2}$.

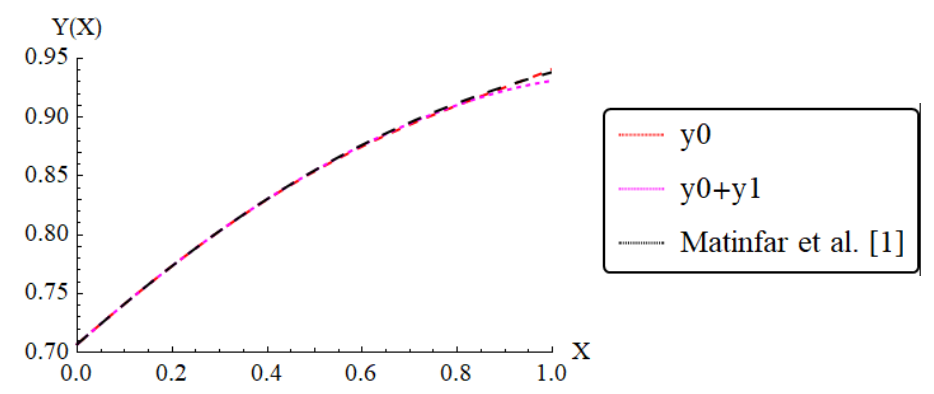

Fig. 1: Approximate and exact solutions for Example 1 
Table 1: Relative errors for example 1

\begin{tabular}{cccccc}
\hline $\mathbf{x}$ & HPM 2 terms & HPM 3 terms & Matinfaret al. [1] & HPM 2 - Error & HPM 3 - Error \\
\hline 0.0 & $7.07 \mathrm{E}-01$ & $7.07 \mathrm{E}-01$ & $7.07 \mathrm{E}-01$ & $1.11 \mathrm{E}-16$ & $1.11 \mathrm{E}-16$ \\
\hline 0.1 & $7.42 \mathrm{E}-01$ & $7.42 \mathrm{E}-01$ & $7.42 \mathrm{E}-01$ & $1.66 \mathrm{E}-06$ & $4.50 \mathrm{E}-07$ \\
\hline 0.2 & $7.74 \mathrm{E}-01$ & $7.74 \mathrm{E}-01$ & $7.74 \mathrm{E}-01$ & $2.59 \mathrm{E}-05$ & $6.49 \mathrm{E}-06$ \\
\hline 0.3 & $8.03 \mathrm{E}-01$ & $8.03 \mathrm{E}-01$ & $8.04 \mathrm{E}-01$ & $1.24 \mathrm{E}-04$ & $2.91 \mathrm{E}-05$ \\
\hline 0.4 & $8.30 \mathrm{E}-01$ & $8.31 \mathrm{E}-01$ & $8.31 \mathrm{E}-01$ & $3.61 \mathrm{E}-04$ & $8.15 \mathrm{E}-05$ \\
\hline 0.5 & $8.54 \mathrm{E}-01$ & $8.55 \mathrm{E}-01$ & $8.55 \mathrm{E}-01$ & $7.74 \mathrm{E}-04$ & $1.82 \mathrm{E}-04$ \\
\hline 0.6 & $8.75 \mathrm{E}-01$ & $8.76 \mathrm{E}-01$ & $8.77 \mathrm{E}-01$ & $1.33 \mathrm{E}-03$ & $3.76 \mathrm{E}-04$ \\
\hline 0.7 & $8.94 \mathrm{E}-01$ & $8.95 \mathrm{E}-01$ & $8.96 \mathrm{E}-01$ & $1.84 \mathrm{E}-03$ & $7.80 \mathrm{E}-04$ \\
\hline 0.8 & $9.10 \mathrm{E}-01$ & $9.10 \mathrm{E}-01$ & $9.12 \mathrm{E}-01$ & $1.91 \mathrm{E}-03$ & $1.66 \mathrm{E}-03$ \\
\hline 0.9 & $9.26 \mathrm{E}-01$ & $9.23 \mathrm{E}-01$ & $9.26 \mathrm{E}-01$ & $8.34 \mathrm{E}-04$ & $3.55 \mathrm{E}-03$ \\
\hline 1.0 & $9.41 \mathrm{E}-01$ & $9.31 \mathrm{E}-01$ & $9.39 \mathrm{E}-01$ & $2.48 \mathrm{E}-03$ & $7.36 \mathrm{E}-03$ \\
\hline
\end{tabular}

Example 2:

$$
\begin{gathered}
y^{\prime \prime}(x)+p y(x)+q y^{3}(x)+r y^{5}(x)=0 . \\
y(0)=\sqrt{\frac{K}{2+D}}, \quad y^{\prime}(0)=0
\end{gathered}
$$

where, $K=4 \sqrt{\frac{3 p^{2}}{3 q^{2}-16 r p}}$ and $D=-1+\frac{\sqrt{3} q}{\sqrt{3 q^{2}-16 r p}}$.

Let us consider, $r=3, p=-1, q=4$, then the exact solution [1] is

$$
y(x)=\sqrt{\frac{K \operatorname{sech}^{2}(\sqrt{-p} x)}{2+D \operatorname{sech}^{2}(\sqrt{-p} x)}}
$$

Using the same approach we get,

$$
\begin{aligned}
& y_{0}=\frac{2^{\frac{1}{4}}}{\sqrt{2+\sqrt{2}}} \\
& y_{1}=2^{\frac{1}{4}} \sqrt{2+\sqrt{2}}(3+2 \sqrt{2}) e^{-x}\left(-1+e^{x}\right)^{2} \text {. } \\
& y_{2}=-\frac{3 \times 2^{\frac{1}{4}}(-11+6 \sqrt{2}) e^{-x}\left(-1+e^{x}\right)\left(2+e^{x}(-2+x)+x\right)}{(\sqrt{2+\sqrt{2}})^{\frac{3}{2}}} .
\end{aligned}
$$




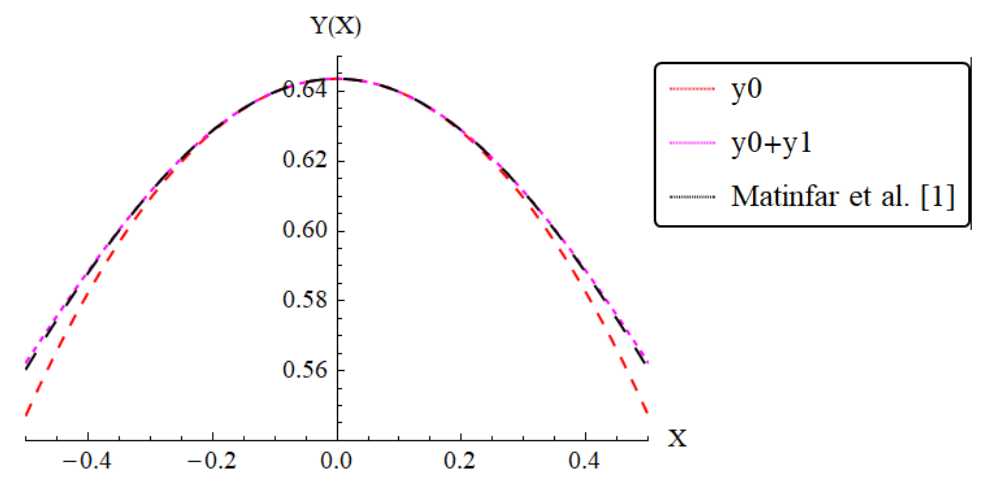

Fig. 2: Approximate and exact solutions for Example 2

Table 2: Relative Errors for example 2

\begin{tabular}{cccccc}
\hline $\mathbf{x}$ & HPM 2 & HPM 3 & Matinfar et al. [1] & HPM 2 - Error & HPM 3 - Error \\
\hline-0.5 & $5.47 \mathrm{E}-01$ & $5.62 \mathrm{E}-01$ & $5.61 \mathrm{E}-01$ & $1.32 \mathrm{E}-02$ & $1.85 \mathrm{E}-03$ \\
\hline-0.4 & $5.82 \mathrm{E}-01$ & $5.89 \mathrm{E}-01$ & $5.88 \mathrm{E}-01$ & $5.63 \mathrm{E}-03$ & $5.06 \mathrm{E}-04$ \\
\hline-0.3 & $6.09 \mathrm{E}-01$ & $6.11 \mathrm{E}-01$ & $6.11 \mathrm{E}-01$ & $1.84 \mathrm{E}-03$ & $9.32 \mathrm{E}-05$ \\
\hline-0.2 & $6.28 \mathrm{E}-01$ & $6.29 \mathrm{E}-01$ & $6.29 \mathrm{E}-01$ & $3.72 \mathrm{E}-04$ & $8.40 \mathrm{E}-06$ \\
\hline-0.1 & $6.40 \mathrm{E}-01$ & $6.40 \mathrm{E}-01$ & $6.40 \mathrm{E}-01$ & $2.36 \mathrm{E}-05$ & $1.33 \mathrm{E}-07$ \\
\hline 0.0 & $6.44 \mathrm{E}-01$ & $6.44 \mathrm{E}-01$ & $6.44 \mathrm{E}-01$ & $0.0 \mathrm{E}+00$ & $0.0 \mathrm{E}+00$ \\
\hline 0.1 & $6.40 \mathrm{E}-01$ & $6.40 \mathrm{E}-01$ & $6.40 \mathrm{E}-01$ & $2.36 \mathrm{E}-05$ & $1.33 \mathrm{E}-07$ \\
\hline 0.2 & $6.28 \mathrm{E}-01$ & $6.29 \mathrm{E}-01$ & $6.29 \mathrm{E}-01$ & $3.72 \mathrm{E}-04$ & $8.40 \mathrm{E}-06$ \\
\hline 0.3 & $6.09 \mathrm{E}-01$ & $6.11 \mathrm{E}-01$ & $6.11 \mathrm{E}-01$ & $1.84 \mathrm{E}-03$ & $9.32 \mathrm{E}-05$ \\
\hline 0.4 & $5.82 \mathrm{E}-01$ & $5.89 \mathrm{E}-01$ & $5.88 \mathrm{E}-01$ & $5.63 \mathrm{E}-03$ & $5.06 \mathrm{E}-04$ \\
\hline 0.5 & $5.47 \mathrm{E}-01$ & $5.62 \mathrm{E}-01$ & $5.61 \mathrm{E}-01$ & $1.32 \mathrm{E}-02$ & $1.85 \mathrm{E}-03$ \\
\hline
\end{tabular}

From the above findings, it can be seen that there is an insignificant difference between the results of two and three terms HPM solutions and exact solution. It motivates us to continue further studies using different boundary conditions.

\section{Results and Discussion}

Solution of Liénard differential equation with different types of boundary conditions using HPM is presented and discussed.

Example 3: $y^{\prime \prime}(x)+l y(x)+m y^{3}(x)+n y^{5}(x)=0$.

And, boundary conditions (BCs) are,

$$
\begin{gathered}
y(a)=\alpha, \quad y(b)=\beta \\
\text { or, } y(a)=\alpha, y^{\prime}(b)=\beta
\end{gathered}
$$




$$
\begin{aligned}
& \text { or, } y^{\prime}(a)=\alpha, y(b)=\beta \\
& \text { or, } y^{\prime}(a)=\alpha, y^{\prime}(b)=\beta
\end{aligned}
$$

The Homotopy is,

$$
y^{\prime \prime}+l y-\left(y_{0}^{\prime \prime}+l y_{0}\right)+t\left(y_{0}^{\prime \prime}+l y_{0}\right)+t\left[m y^{3}+n y^{5}\right]=0 .
$$

Putting,

$$
\begin{gathered}
y=y_{0}+t y_{1}+t^{2} y_{2} \\
y_{0}=\left(-\left(\frac{b^{2} \alpha-a^{2} \beta}{a b(a-b)}\right) x-\frac{(\beta a-\alpha b)}{a b(a-b)} x^{2}\right)
\end{gathered}
$$

Andusing BCs (I), we get,

$$
y_{0}{ }^{\prime \prime}(x)+l y_{0}(x)-\left(\frac{-2(a \beta-b \alpha)}{a b(a-b)}+l\left(-\left(\frac{b^{2} \alpha-a^{2} \beta}{a b(a-b)}\right) x-\frac{(\beta a-\alpha b)}{a b(a-b)} x^{2}\right)\right)=0
$$

with

$$
\begin{gathered}
y_{0}(a)=\alpha, \quad y_{0}(b)=\beta \\
y_{1}^{\prime \prime}(x)+l y_{1}(x)-\left(\frac{-2(a \beta-b \alpha)}{a b(a-b)}+l\left(-\left(\frac{b^{2} \alpha-a^{2} \beta}{a b(a-b)}\right) x-\frac{(\beta a-\alpha b)}{a b(a-b)} x^{2}\right)\right)+m y_{0}^{3}+n y_{0}^{5} \\
=0
\end{gathered}
$$

with

$$
\begin{gathered}
y_{1}(a)=0, \quad y_{1}(b)=0 \\
y_{2}^{\prime \prime}+l y_{2}+3 m y_{0}^{3} y_{1}+5 n y_{0}^{5} y_{1}=0 w i t h y_{2}(a)=0, \quad y_{2}(b)=0
\end{gathered}
$$

Let us consider, $n=-3, l=-1, m=4, \alpha=0, \beta=1, a=-2, b=2$. Solving the above differential equationsusing MATHEMATICA, we get,

$$
\begin{aligned}
& y_{0}=-\left(\frac{1}{8}\right)(-2-x) x \\
& y_{1}=\frac{1}{32768\left(-1+e^{8}\right)} e^{-x}\left(-102082560 e^{2}+15639552 e^{6}-15639552 e^{2+2 x}\right. \\
& +102082560 e^{6+2 x}+e^{x}\left(15639552+11919104 x+7823872 x^{2}+1985152 x^{3}\right. \\
& \left.+651648 x^{4}+99360 x^{5}+21824 x^{6}+2400 x^{7}+390 x^{8}+30 x^{9}+3 x^{10}\right)-e^{8+x}(15639552 \\
& +11919104 x+7823872 x^{2}+1985152 x^{3}+651648 x^{4}+99360 x^{5}+21824 x^{6} \\
& \left.\left.+2400 x^{7}+390 x^{8}+30 x^{9}+3 x^{10}\right)\right)
\end{aligned}
$$




$$
\begin{aligned}
& y_{2}=\left(2.7 \times 10^{-19}\right) \mathrm{e}^{-\mathrm{x}}\left(( - 3 . 8 \times 1 0 ^ { 2 } ) \mathrm { e } ^ { 2 ( 5 + \mathrm { x } ) } \left(-2.1 \times 10^{201}+\left(4.1 \times 10^{10}\right) \mathrm{x}-(4.1 \times\right.\right. \\
& \left.10^{10}\right) \mathrm{x}^{2}+\left(2.7 \times 10^{10}\right) \mathrm{x}^{3}-\left(1.4 \times 10^{10}\right) \mathrm{x}^{4}+\left(6.4 \times 10^{8}\right) \mathrm{x}^{5}-\left(5.0 \times 10^{9}\right) \mathrm{x}^{6}-(1.0 \times \\
& \left.\left.10^{8}\right) \mathrm{x}^{7}+\left(1.5 \times 10^{8}\right) \mathrm{x}^{8}+\left(2.3 \times 10^{8}\right) \mathrm{x}^{9}+\left(5.9 \times 10^{7}\right) \mathrm{x}^{10}+\left(1.1 \times 10^{7}\right) \mathrm{x}^{11}\right)+(3.8 \times \\
& \left.10^{2}\right) \mathrm{e}^{2+2 \mathrm{x}}\left(-2.1 \times 10^{20}+\left(4.1 \times 10^{10}\right) \mathrm{x}-\left(4.1 \times 10^{10}\right) \mathrm{x}^{2}+\left(2.7 \times 10^{10}\right) \mathrm{x}^{3}-(1.4 \times\right. \\
& \left.10^{10}\right) \mathrm{x}^{4}+\left(6.4 \times 10^{8}\right) \mathrm{x}^{5}-\left(5.0 \times 10^{9}\right) \mathrm{x}^{6}-\left(1.0 \times 10^{8}\right) \mathrm{x}^{7}+\left(1.5 \times 10^{8}\right) \mathrm{x}^{8}+\left(2.3 \times 10^{8}\right) \mathrm{x}^{9}+ \\
& \left.\left(5.9 \times 10^{7}\right) x^{10}+\left(1.1 \times 10^{7}\right) x^{11}\right)-\left(4.6 \times 10^{8}\right)\left(2.1 \times 10^{20}+\left(2.1 \times 10^{12}\right) x+(2.1 \times\right. \\
& \left.10^{12}\right) \mathrm{x}^{2}+\left(1.4 \times 10^{12}\right) \mathrm{x}^{3}+\left(7.1 \times 10^{11}\right) \mathrm{x}^{4}+\left(2.9 \times 10^{11}\right) \mathrm{x}^{5}+\left(1.0 \times 10^{11}\right) \mathrm{x}^{6}+(3.0 \times \\
& \left.\left.10^{10}\right) \mathrm{x}^{7}+\left(7.5 \times 10^{9}\right) \mathrm{x}^{8}+\left(1.4 \times 10^{9}\right) \mathrm{x}^{9}+\left(1.8 \times 10^{8}\right) \mathrm{x}^{10}+\left(1.1 \times 10^{7}\right) \mathrm{x}^{11}\right)+(1.3 \times \\
& \left.10^{3}\right) \mathrm{e}^{2(7+\mathrm{x})}\left(-4.1 \times 10^{20}+\left(8.0 \times 10^{10}\right) \mathrm{x}-\left(8.0 \times 10^{10}\right) \mathrm{x}^{2}+\left(5.3 \times 10^{10}\right) \mathrm{x}^{3}-(2.7 \times\right. \\
& \left.10^{10}\right) \mathrm{x}^{4}+\left(1.2 \times 10^{9}\right) \mathrm{x}^{5}-\left(9.8 \times 10^{9}\right) \mathrm{x}^{6}-\left(2.0 \times 10^{8}\right) \mathrm{x}^{7}+\left(2.9 \times 10^{8}\right) \mathrm{x}^{8}+\left(4.5 \times 10^{8}\right) \mathrm{x}^{9}+ \\
& \left.\left(1.2 \times 10^{8}\right) \mathrm{x}^{10}+\left(2.1 \times 10^{7}\right) \mathrm{x}^{11}\right)-\left(1.3 \times 10^{3}\right) \mathrm{e}^{6+2 . \mathrm{x}}\left(-4.1 \times 10^{20}+\left(8.0 \times 10^{10}\right) \mathrm{x}-\right. \\
& \left(8.0 \times 10^{10}\right) \mathrm{x}^{2}+\left(5.3 \times 10^{10}\right) \mathrm{x}^{3}-\left(2.7 \times 10^{10}\right) \mathrm{x}^{4}+\left(1.2 \times 10^{9}\right) \mathrm{x}^{5}-\left(9.8 \times 10^{9}\right) \mathrm{x}^{6}- \\
& \left.\left(2.0 \times 10^{8}\right) \mathrm{x}^{7}+\left(2.9 \times 10^{8}\right) \mathrm{x}^{8}+\left(4.5 \times 10^{8}\right) \mathrm{x}^{9}+\left(1.2 \times 10^{8}\right) \mathrm{x}^{10}+\left(2.1 \times 10^{7}\right) \mathrm{x}^{11}\right)+(2.8 \times \\
& \left.10^{7}\right)\left(4.1 \times 10^{20}+\left(4.2 \times 10^{12}\right) \mathrm{x}+\left(4.2 \times 10^{12}\right) \mathrm{x}^{2}+\left(2.8 \times 10^{12}\right) \mathrm{x}^{3}+\left(1.4 \times 10^{12}\right) \mathrm{x}^{4}+\right. \\
& \left(5.7 \times 10^{11}\right) \mathrm{x}^{5}+\left(2.0 \times 10^{11}\right) \mathrm{x}^{6}+\left(6.0 \times 10^{10}\right) \mathrm{x}^{7}+\left(1.5 \times 10^{10}\right) \mathrm{x}^{8}+\left(2.8 \times 10^{9}\right) \mathrm{x}^{9}+ \\
& \left.\left(3.5 \times 10^{8}\right) \mathrm{x}^{10}+\left(2.1 \times 10^{7}\right) \mathrm{x}^{11}\right)+\left(1.2 \times 10^{3}\right) \mathrm{e}^{\mathrm{x}}\left(7.0 \times 10^{19}+\left(5.4 \times 10^{19}\right) \mathrm{x}+\right. \\
& \left(3.5 \times 10^{19}\right) \mathrm{x}^{2}+\left(8.9 \times 10^{18}\right) \mathrm{x}^{3}+\left(2.9 \times 10^{18}\right) \mathrm{x}^{4}+\left(4.5 \times 10^{17}\right) \mathrm{x}^{5}+\left(9.8 \times 10^{16}\right) \mathrm{x}^{6}+ \\
& \left(1.1 \times 10^{16}\right) x^{7}+\left(1.7 \times 10^{15}\right) x^{8}+\left(1.5 \times 10^{14}\right) x^{9}+\left(1.9 \times 10^{13}\right) x^{10}+\left(1.3 \times 10^{12}\right) x^{11}+ \\
& \left(1.5 \times 10^{11}\right) x^{12}+\left(8.6 \times 10^{9}\right) x^{13}+\left(8.1 \times 10^{8}\right) x^{14}+\left(4.1 \times 10^{7}\right) x^{15}+\left(3.3 \times 10^{6}\right) x^{16}+ \\
& \left.\left(1.4 \times 10^{5}\right) x^{17}+\left(9.8 \times 10^{3}\right) x^{18}+\left(3.0 \times 10^{2}\right) x^{19}+\left(1.5 \times 10^{1}\right) x^{20}\right)- \\
& \left(2.3 \times 10^{3}\right) \mathrm{e}^{8+\mathrm{x}}\left(7.0 \times 10^{19}+\left(5.4 \times 10^{19}\right) \mathrm{x}+\left(3.5 \times 10^{19}\right) \mathrm{x}^{2}+\left(8.9 \times 10^{18}\right) \mathrm{x}^{3}+\right. \\
& \left(2.9 \times 10^{18}\right) \mathrm{x}^{4}+\left(4.5 \times 10^{17}\right) \mathrm{x}^{5}+\left(9.8 \times 10^{16}\right) \mathrm{x}^{6}+\left(1.1 \times 10^{16}\right) \mathrm{x}^{7}+\left(1.7 \times 10^{15}\right) \mathrm{x}^{8}+ \\
& \left(1.5 \times 10^{14}\right) \mathrm{x}^{9}+\left(1.9 \times 10^{13}\right) \mathrm{x}^{10}+\left(1.3 \times 10^{12}\right) \mathrm{x}^{11}+\left(1.5 \times 10^{11}\right) \mathrm{x}^{12}+\left(8.6 \times 10^{9}\right) \mathrm{x}^{13}+ \\
& \left(8.1 \times 10^{8}\right) \mathrm{x}^{14}+\left(4.1 \times 10^{7}\right) \mathrm{x}^{15}+\left(3.3 \times 10^{6}\right) \mathrm{x}^{16}+\left(1.4 \times 10^{5}\right) \mathrm{x}^{17}+\left(9.8 \times 10^{3}\right) \mathrm{x}^{18}+ \\
& \left.\left(3.0 \times 10^{2}\right) \mathrm{x}^{19}+\left(1.5 \times 10^{1}\right) \mathrm{x}^{20}\right)+\left(1.2 \times 10^{3}\right)\left(7.0 \times 10^{19}+\left(5.4 \times 10^{19}\right) \mathrm{x}+(3.5 \times\right. \\
& \left.10^{19}\right) \mathrm{x}^{2}+\left(8.9 \times 10^{18}\right) \mathrm{x}^{3}+\left(2.9 \times 10^{18}\right) \mathrm{x}^{4}+\left(4.5 \times 10^{17}\right) \mathrm{x}^{5}+\left(9.8 \times 10^{16}\right) \mathrm{x}^{6}+(1.1 \times \\
& \left.10^{16}\right) x^{7}+\left(1.7 \times 10^{15}\right) x^{8}+\left(1.5 \times 10^{14}\right) x^{9}+\left(1.9 \times 10^{13}\right) x^{10}+\left(1.3 \times 10^{12}\right) x^{11}+ \\
& \left(1.5 \times 10^{11}\right) x^{12}+\left(8.6 \times 10^{9}\right) x^{13}+\left(8.1 \times 10^{8}\right) x^{14}+\left(4.1 \times 10^{7}\right) x^{15}+\left(3.3 \times 10^{6}\right) x^{16}+ \\
& \left(1.4 \times 10^{5}\right) \mathrm{x}^{17}+\left(9.8 \times 10^{3}\right) \mathrm{x}^{18}+\left(3.0 \times 10^{2}\right) \mathrm{x}^{19}+\left(1.5 \times 10^{1}\right) \mathrm{x}^{20}
\end{aligned}
$$

Similarly using BCs. (II) and $m=4, l=-1, n=-3, \alpha=0, \beta=1, a=-2, b=2$, we get,

$$
y_{0}=-\left(\frac{1}{12}\right)(-4-x) x
$$


$\mathrm{y}_{1}=\frac{1}{82944\left(1+\mathrm{e}^{8}\right)} \mathrm{e}^{-\mathrm{x}}\left(77352192 \mathrm{e}^{2}+77352192 \mathrm{e}^{6+2 \mathrm{x}}-\mathrm{e}^{\mathrm{x}}\left(10642176+10283520 \mathrm{x}+532800 \mathrm{x}^{2}+\right.\right.$ $1709312 \times 3+443424 \mathrm{x} 4+86080 \mathrm{x} 5+15088 \mathrm{x} 6+2080 \mathrm{x} 7+250 \mathrm{x} 8+20 \mathrm{x} 9+\mathrm{x} 10)-\mathrm{e} 8+\mathrm{x}$ $\left(10642176+10283520 x+5328000 x^{2}+17093212 x^{3}+443424 x^{4}+86080 x^{5}+15088 x^{6}+2080 x^{7}+\right.$ $\left.\left.250 \mathrm{x}^{8}+20 \mathrm{x}^{9}+\mathrm{x}^{10}\right)\right)$

$$
\begin{aligned}
& y_{2}=3.0 \times 10^{-19} e^{-x}\left(-9.3 \times 10^{26}+\left(7.7 \times 10^{20}\right) e^{2+5 x}+\left(7.7 \times 10^{20}\right) e^{2+2 x}\right)+(9.6 \\
& \times 10) \mathrm{e}^{2.0(7.0+\mathrm{x})}\left(-2.2 \times 10^{20}+\left(1.2 \times 10^{11}\right) \mathrm{x}-\left(1.2 \times 10^{11}\right) \mathrm{x}^{2}\right. \\
& +\left(7.8 \times 10^{10}\right) \mathrm{x}^{3}-\left(3.9 \times 10^{10}\right) \mathrm{x}^{4}-\left(6.6 \times 10^{10}\right) \mathrm{x}^{5}-\left(1.9 \times 10^{10}\right) \mathrm{x}^{6} \\
& +\left(3.5 \times 10^{9}\right) x^{7}+\left(3.7 \times 10^{9}\right) x^{8}+\left(1.1 \times 10^{9}\right) x^{9}+\left(1.7 \times 10^{8}\right) x^{10} \\
& \left.+\left(1.0 \times 10^{7}\right) \mathrm{x}^{11}\right)-\left(9.6 \times 10^{1}\right) \mathrm{e}^{6.0+2.0 \times}\left(2.2 \times 10^{20}+\left(1.2 \times 10^{11}\right) \mathrm{x}-(1.2\right. \\
& \left.\times 10^{11}\right) \mathrm{x}^{2}+\left(7.8 \times 10^{10}\right) \mathrm{x}^{3}-\left(3.9 \times 10^{10}\right) \mathrm{x}^{4}-\left(6.6 \times 10^{10}\right) \mathrm{x}^{5}-(1.9 \\
& \left.\times 10^{10}\right) \mathrm{x}^{6}+\left(3.5 \times 10^{9}\right) \mathrm{x}^{7}+\left(3.7 \times 10^{9}\right) \mathrm{x}^{8}+\left(1.1 \times 10^{9}\right) \mathrm{x}^{9}+\left(1.7 \times 10^{8}\right) \mathrm{x}^{10} \\
& \left.+\left(1.0 \times 10^{7}\right) \mathrm{x}^{11}\right)-\left(2.1 \times 10^{6}\right)\left(-2.2 \times 1020+\left(7.3 \times 10^{12}\right) \mathrm{x}+(7.3\right. \\
& \left.\times 10^{12}\right) x^{2}+\left(4.9 \times 10^{12}\right) x^{3}+\left(2.4 \times 10^{12}\right) x^{4}+\left(1.1 \times 10^{12}\right) x^{5}+(3.9 \\
& \left.\times 10^{11}\right) x^{6}+\left(1.1 \times 10^{11}\right) x^{7}+\left(2.4 \times 10^{10}\right) x^{8}+\left(3.4 \times 10^{9}\right) x^{9}+(2.8 \\
& \left.\left.\times 10^{8}\right) \mathrm{x}^{10}+\left(1.0 \times 10^{7}\right) \mathrm{x}^{11}\right)+\left(7.1 \times 10^{2}\right)\left(2.2 \times 10^{20}+\left(7.3 \times 10^{12}\right) \mathrm{x}\right. \\
& +\left(7.3 \times 10^{12}\right) \mathrm{x}^{2}+\left(4.9 \times 10^{12}\right) \mathrm{x}^{3}+\left(2.4 \times 10^{12}\right) \mathrm{x}^{4}+\left(1.1 \times 10^{12}\right) \mathrm{x}^{5} \\
& +\left(3.9 \times 10^{11}\right) x^{6}+\left(1.1 \times 10^{11}\right) x^{7}+\left(2.4 \times 10^{10}\right) x^{8}+\left(3.4 \times 10^{9}\right) x^{9} \\
& \left.+\left(2.8 \times 10^{8}\right) \mathrm{x}^{10}+\left(1.0 \times 10^{7}\right) \mathrm{x}^{11}\right) \\
& -\left(5.5 \times 10^{1}\right) \mathrm{e}^{x}\left(5.3 \times 10^{19}+\left(5.1 \times 10^{19}\right) \mathrm{x}+\left(2.6 \times 10^{19}\right) \mathrm{x}^{2}\right. \\
& +\left(8.5 \times 10^{18}\right) x^{3}+\left(2.2 \times 10^{18}\right) x^{4}+\left(4.2 \times 10^{17}\right) x^{5}+\left(7.3 \times 10^{16}\right) x^{6} \\
& +\left(1.0 \times 10^{16}\right) x^{7}+\left(1.3 \times 10^{15}\right) x^{8}+\left(1.4 \times 10^{14}\right) x^{9}+\left(1.5 \times 10^{13}\right) x^{10} \\
& +\left(1.3 \times 10^{12}\right) x^{11}+\left(1.1 \times 10^{11}\right) x^{12}+\left(8.2 \times 10^{9}\right) x^{13}+\left(6.0 \times 10^{8}\right) x^{14} \\
& +\left(3.8 \times 10^{7}\right) x^{15}+\left(2.4 \times 10^{6}\right) x^{16}+\left(1.2 \times 10^{5}\right) x^{17}+\left(5.9 \times 10^{3}\right) x^{18} \\
& \left.+\left(2.0 \times 10^{2}\right) \mathrm{x}^{19}+5.0 \mathrm{x}^{20}\right)+(5.5 \times 10) \mathrm{e}^{16+\mathrm{x}}\left(5.3 \times 10^{19}+\left(5.1 \times 10^{19}\right) \mathrm{x}\right. \\
& +\left(2.6 \times 10^{19}\right) x^{2}+\left(8.5 \times 10^{18}\right) x^{3}+\left(2.2 \times 10^{18}\right) x^{4}+\left(4.2 \times 10^{17}\right) x^{5}+(7.3 \\
& \left.\times 10^{16}\right) x^{6}+\left(1.0 \times 10^{16}\right) x^{7}+\left(1.3 \times 10^{15}\right) x^{8}+\left(1.4 \times 10^{14}\right) x^{9}+(1.5 \\
& \left.\times 10^{13}\right) x^{10}+\left(1.3 \times 10^{12}\right) x^{11}+\left(1.1 \times 10^{11}\right) x^{12}+\left(8.2 \times 10^{9}\right) x^{13}+(6.0 \\
& \left.\times 10^{8}\right) x^{14}+\left(3.8 \times 10^{7}\right) x^{15}+\left(2.4 \times 10^{6}\right) x^{16}+\left(1.2 \times 10^{5}\right) x^{17}+(5.9 \\
& \left.\left.\left.\times 10^{3}\right) \mathrm{x}^{18}+\left(2.0 \times 10^{2}\right) \mathrm{x}^{19}+5.0 \mathrm{x}^{20}\right)\right)
\end{aligned}
$$

Similarly usingBCs. (III) and $l=-1, m=4, n=-3, \alpha=1, \beta=0, a=-2, b=2$ we get,

$y_{0}=\frac{1}{12}(-4+x) x$ 


$$
\begin{aligned}
& y_{1}=\frac{1}{82944\left(1+\mathrm{e}^{8}\right)} \mathrm{e}^{-\mathrm{x}}\left(77352192 \mathrm{e}^{6}+77352192 \mathrm{e}^{2+2 \mathrm{x}}-\mathrm{e}^{\mathrm{x}}(10642176-10283520 \mathrm{x}\right. \\
& +5328000 x^{2}-1709312 x^{3}+443424 x^{4}-86080 x^{5}+15088 x^{6}-2080 x^{7}+250 x^{8}-20 x^{9} \\
& \left.+\mathrm{x}^{10}\right)-\mathrm{e}^{8+\mathrm{x}}\left(10642176-10283520 \mathrm{x}+5328000 \mathrm{x}^{2}-1709312 \mathrm{x}^{3}+443424 \mathrm{x}^{4}-86080 \mathrm{x}^{5}\right. \\
& \left.\left.+15088 \mathrm{x}^{6}-2080 \mathrm{x}^{7}+250 \mathrm{x}^{8}-20 \mathrm{x}^{9}+\mathrm{x}^{10}\right)\right) \\
& y 2=\left(3.0 \times 10^{-19}\right) \mathrm{e}^{-\mathrm{x}}\left(( 9 . 6 \times 1 0 ^ { 1 } ) \mathrm { e } ^ { 2 ( 5 + \mathrm { x } ) } \left(-2.2 \times 10^{20}+\left(7.3 \times 10^{12}\right) \mathrm{x}-(7.3 \times\right.\right. \\
& \left.10^{12}\right) \mathrm{x}^{2}+\left(4.9 \times 10^{12}\right) \mathrm{x}^{3}-\left(2.4 \times 10^{12}\right) \mathrm{x}^{4}+\left(1.1 \times 10^{12}\right) \mathrm{x}^{5}-\left(3.9 \times 10^{11}\right) \mathrm{x}^{6}+(1.1 \times \\
& \left.\left.10^{11}\right) \mathrm{x}^{7}-\left(2.4 \times 10^{10}\right) \mathrm{x}^{8}+\left(3.4 \times 10^{9}\right) \mathrm{x}^{9}-\left(2.8 \times 10^{8}\right) \mathrm{x}^{10}+\left(1.0 \times 10^{7}\right) \mathrm{x}^{11}\right)+(9.6 \times \\
& \left.10^{1}\right) \mathrm{e}^{2+2 \mathrm{x}}\left(-2.2 \times 10^{20}+\left(7.3 \times 10^{12}\right) \mathrm{x}-\left(7.3 \times 10^{12}\right) \mathrm{x}^{2}+\left(4.9 \times 10^{12}\right) \mathrm{x}^{3}-(2.4 \times\right. \\
& \left.10^{12}\right) \mathrm{x}^{4}+\left(1.1 \times 10^{12}\right) \mathrm{x}^{5}-\left(3.9 \times 10^{11}\right) \mathrm{x}^{6}+\left(1.1 \times 10^{11}\right) \mathrm{x}^{7}-\left(2.4 \times 10^{10}\right) \mathrm{x}^{8}+(3.4 \times \\
& \left.\left.10^{9}\right) \mathrm{x}^{9}-\left(2.8 \times 10^{8}\right) \mathrm{x}^{10}+\left(1.0 \times 10^{7}\right) \mathrm{x}^{11}\right)-\left(1.2 \times 10^{8}\right)\left(2.2 \times 10^{20}+\left(1.2 \times 10^{11}\right) \mathrm{x}+\right. \\
& \left(1.2 \times 10^{11}\right) \mathrm{x}^{2}+\left(7.8 \times 10^{10}\right) \mathrm{x}^{3}+\left(3.9 \times 10^{10}\right) \mathrm{x}^{4}-\left(6.6 \times 10^{10}\right) \mathrm{x}^{5}+\left(1.9 \times 10^{10}\right) \mathrm{x}^{6}+ \\
& \left.\left(3.5 \times 10^{9}\right) x^{7}-\left(3.7 \times 10^{9}\right) x^{8}+\left(1.1 \times 10^{9}\right) x^{9}-\left(1.7 \times 10^{8}\right) x^{10}+\left(1.0 \times 10^{7}\right) x^{11}\right)+ \\
& \left(5.5 \times 10^{1}\right) \mathrm{e}^{\mathrm{x}}\left(5.3 \times 10^{19}-\left(5.1 \times 10^{19}\right) \mathrm{x}+\left(2.6 \times 10^{19}\right) \mathrm{x}^{2}-\left(8.5 \times 10^{18}\right) \mathrm{x}^{3}+(2.2 \times\right. \\
& \left.10^{18}\right) \mathrm{x}^{4}-\left(4.2 \times 10^{17}\right) \mathrm{x}^{5}+\left(7.3 \times 10^{16}\right) \mathrm{x}^{6}-\left(1.0 \times 10^{16}\right) \mathrm{x}^{7}+\left(1.3 \times 10^{15}\right) \mathrm{x}^{8}-(1.4 \times \\
& \left.10^{14}\right) \mathrm{x}^{9}+\left(1.5 \times 10^{13}\right) \mathrm{x}^{10}-\left(1.3 \times 10^{12}\right) \mathrm{x}^{11}+\left(1.1 \times 10^{11}\right) \mathrm{x}^{12}-\left(8.2 \times 10^{9}\right) \mathrm{x}^{13}+ \\
& \left(6.0 \times 10^{8}\right) x^{14}-\left(3.8 \times 10^{7}\right) x^{15}+\left(2.4 \times 10^{6}\right) x^{16}-\left(1.2 \times 10^{5}\right) x^{17}+\left(5.9 \times 10^{3}\right) x^{18}- \\
& \left.\left(2.0 \times 10^{2}\right) \mathrm{x}^{19}+5.0 \mathrm{x}^{20}\right)+\left(1.1 \times 10^{2}\right) \mathrm{e}^{8+\mathrm{x}}\left(5.3 \times 10^{19}-\left(5.1 \times 10^{19}\right) \mathrm{x}+\left(2.6 \times 10^{19}\right) \mathrm{x}^{2}-\right. \\
& \left(8.5 \times 10^{18}\right) \mathrm{x}^{3}+\left(2.2 \times 10^{18}\right) \mathrm{x}^{4}-\left(4.2 \times 10^{17}\right) \mathrm{x}^{5}+\left(7.3 \times 10^{16}\right) \mathrm{x}^{6}-\left(1.0 \times 10^{16}\right) \mathrm{x}^{7}+ \\
& \left(1.3 \times 10^{15}\right) x^{8}-\left(1.4 \times 10^{14}\right) x^{9}+\left(1.5 \times 10^{13}\right) x^{10}-\left(1.3 \times 10^{12}\right) x^{11}+\left(1.1 \times 10^{11}\right) x^{12}- \\
& \left(8.2 \times 10^{9}\right) \mathrm{x}^{13}+\left(6.0 \times 10^{8}\right) \mathrm{x}^{14}-\left(3.8 \times 10^{7}\right) \mathrm{x}^{15}+\left(2.4 \times 10^{6}\right) \mathrm{x}^{16}-\left(1.2 \times 10^{5}\right) \mathrm{x}^{17}+ \\
& \left.\left(5.9 \times 10^{3}\right) \mathrm{x}^{18}-\left(2.0 \times 10^{2}\right) \mathrm{x}^{19}+5.0 \mathrm{x}^{20}\right)+\left(5.5 \times 10^{1}\right) \mathrm{e}^{1.6 \times 10^{1}+\mathrm{x}}\left(5.3 \times 10^{19}-(5.1 \times\right. \\
& \left.10^{19}\right) \mathrm{x}+\left(2.6 \times 10^{19}\right) \mathrm{x}^{2}-\left(8.5 \times 10^{18}\right) \mathrm{x}^{3}+\left(2.2 \times 10^{18}\right) \mathrm{x}^{4}-\left(4.2 \times 10^{17}\right) \mathrm{x}^{5}+(7.3 \times \\
& \left.10^{16}\right) x^{6}-\left(1.0 \times 10^{16}\right) x^{7}+\left(1.3 \times 10^{15}\right) x^{8}-\left(1.4 \times 10^{14}\right) x^{9}+\left(1.5 \times 10^{13}\right) x^{10}- \\
& \left(1.3 \times 10^{12}\right) \mathrm{x}^{11}+\left(1.1 \times 10^{11}\right) \mathrm{x}^{12}-\left(8.2 \times 10^{9}\right) \mathrm{x}^{13}+\left(6.0 \times 10^{8}\right) \mathrm{x}^{14}-\left(3.8 \times 10^{7}\right) \mathrm{x}^{15}+ \\
& \left.\left.\left(2.4 \times 10^{6}\right) \mathrm{x}^{16}-\left(1.2 \times 10^{5}\right) \mathrm{x}^{17}+\left(5.9 \times 10^{3}\right) \mathrm{x}^{18}-\left(2.0 \times 10^{2}\right) \mathrm{x}^{19}+5.0 \mathrm{x}^{20}\right)\right)
\end{aligned}
$$

Similarly using BCs. (IV), and $\alpha=1, \beta=0, a=-2, b=2, m=4, n=-3, l=-1$, we get, $y_{0}=-\left(\frac{1}{8}\right)(-4+x) x$ 


$$
\begin{aligned}
& y_{1}=\frac{1}{32768\left(-1+\mathrm{e}^{8}\right)} \mathrm{e}^{-\mathrm{x}}\left(-235940864 \mathrm{e}^{6}-235940864 \mathrm{e}^{2+2 \mathrm{x}}+\mathrm{e}^{\mathrm{x}}(-32525568\right. \\
& +31367680 \mathrm{x}-16266880 \mathrm{x}^{2}+5225216 \mathrm{x}^{3}-1355232 \mathrm{x}^{4}+262080 \mathrm{x}^{5}-45584 \mathrm{x}^{6}+6240 \mathrm{x}^{7} \\
& \left.-750 \mathrm{x}^{8}+60 \mathrm{x}^{9}-3 \mathrm{x}^{10}\right)+\mathrm{e}^{8+\mathrm{x}}\left(32525568-31367680 \mathrm{x}+16266880 \mathrm{x}^{2}-5225216 \mathrm{x}^{3}\right. \\
& \left.+1355232 \mathrm{x}^{4}-262080 \mathrm{x}^{5}+45584 \mathrm{x}^{6}-6240 \mathrm{x}^{7}+750 \mathrm{x}^{8}-60 \mathrm{x}^{9}+3 \mathrm{x}^{10}\right) \text { ) } \\
& \mathrm{y}_{2}=\left(9.1 \times 10^{-20}\right) e^{-1.0 x}( \\
& \left(-1.3 \times 10^{2}\right) e^{2}+2.0 x\left(-3.1 \times 10^{22}+\left(1.2 \times 10^{15}\right) x-\left(1.2 \times 10^{15}\right) x^{2}+\left(8.2 \times 10^{14}\right) x^{3}\right. \\
& -\left(4.1 \times 10^{14}\right) x^{4}+\left(1.7 \times 10^{14}\right) x^{5}-\left(5.9 \times 10^{13}\right) x^{6}+\left(1.7 \times 10^{13}\right) x^{7} \\
& \left.-\left(3.5 \times 10^{12}\right) x^{8}+\left(4.8 \times 10^{11}\right) x^{9}-\left(4.0 \times 10^{10}\right) x^{10}+\left(1.5 \times 10^{9}\right) x^{11}\right) \\
& +\left(1.3 \times 10^{2}\right) e^{2.0(5.0+x)}\left(-3.1 \times 10^{22}+\left(1.2 \times 10^{15}\right) x-\left(1.2 \times 10^{15}\right) x^{2}+\left(8.2 \times 10^{14}\right) x^{3}\right. \\
& -\left(4.1 \times 10^{14}\right) x^{4}+\left(1.7 \times 10^{14}\right) x^{5}-\left(5.9 \times 10^{13}\right) x^{6}+\left(1.7 \times 10^{13}\right) x^{7} \\
& \left.-\left(3.5 \times 10^{12}\right) x^{8}+\left(4.8 \times 10^{11}\right) x^{9}-\left(4.0 \times 10^{10}\right) x^{10}+\left(1.5 \times 10^{9}\right) x^{11}\right) \\
& -\left(1.5 \times 10^{8}\right)\left(3.1 \times 10^{22}+\left(6.8 \times 10^{12}\right) x+\left(6.8 \times 10^{12}\right) x^{2}+\left(4.6 \times 10^{12}\right) x^{3}+(2.3\right. \\
& \left.\times 10^{12}\right) x^{4}-\left(4.3 \times 10^{12}\right) x^{5}+\left(1.2 \times 10^{12}\right) x^{6}+\left(6.5 \times 10^{11}\right) x^{7}-(5.4 \\
& \left.\left.\times 10^{11}\right) x^{8}+\left(1.6 \times 10^{11}\right) x^{9}-\left(2.4 \times 10^{10}\right) x^{10}+\left(1.5 \times 10^{9}\right) x^{11}\right) \\
& +\left(3.5 \times 10^{3}\right) e^{x}\left(1.6 \times 10^{20}-\left(1.5 \times 10^{20}\right) x+\left(7.9 \times 10^{19}\right) x^{2}-\left(2.6 \times 10^{19}\right) x^{3}+(6.6\right. \\
& \left.\times 10^{18}\right) x^{4}-\left(1.3 \times 10^{18}\right) x^{5}+\left(2.2 \times 10^{17}\right) x^{6}-\left(3.0 \times 10^{16}\right) x^{7}+(3.9 \\
& \left.\times 10^{15}\right) x^{8}-\left(4.2 \times 10^{14}\right) x^{9}+\left(4.4 \times 10^{13}\right) x^{10}-\left(3.8 \times 10^{12}\right) x^{11}+(3.3 \\
& \left.\times 10^{11}\right) x^{12}-\left(2.4 \times 10^{10}\right) x^{13}+\left(1.8 \times 10^{9}\right) x^{14}-\left(1.1 \times 10^{8}\right) x^{15}+(7.1 \\
& \left.\times 10^{6}\right) x^{16}-\left(3.7 \times 10^{5}\right) x^{17}+\left(1.8 \times 10^{4}\right) x^{18}-\left(6.0 \times 10^{2}\right) x^{19}+(1.5 \\
& \left.\left.\times 10^{1}\right) x^{20}\right) \\
& -\left(6.9 \times 10^{3}\right) e^{8+x}\left(1.6 \times 10^{20}-\left(1.5 \times 10^{20}\right) x+\left(7.9 \times 10^{19}\right) x^{2}-\left(2.6 \times 10^{19}\right) x^{3}\right. \\
& +\left(6.6 \times 10^{18}\right) x^{4}-\left(1.3 \times 10^{18}\right) x^{5}+\left(2.2 \times 10^{17}\right) x^{6}-\left(3.0 \times 10^{16}\right) x^{7} \\
& +\left(3.9 \times 10^{15}\right) x^{8}-\left(4.2 \times 10^{14}\right) x^{9}+\left(4.4 \times 10^{13}\right) x^{10}-\left(3.8 \times 10^{12}\right) x^{11} \\
& +\left(3.3 \times 10^{11}\right) x^{12}-\left(2.4 \times 10^{10}\right) x^{13}+\left(1.8 \times 10^{9}\right) x^{14}-\left(1.1 \times 10^{8}\right) x^{15} \\
& +\left(7.1 \times 10^{6}\right) x^{16}-\left(3.7 \times 10^{5}\right) x^{17}+\left(1.8 \times 10^{4}\right) x^{18}-\left(6.0 \times 10^{2}\right) x^{19} \\
& \left.+\left(1.5 \times 10^{1}\right) x^{20}\right) \\
& +\left(3.5 \times 10^{3}\right) e^{16+x}\left(1.6 \times 10^{20}-\left(1.5 \times 10^{20}\right) x+\left(7.9 \times 10^{19}\right) x^{2}-\left(2.6 \times 10^{19}\right) x^{3}\right. \\
& +\left(6.6 \times 10^{18}\right) x^{4}-\left(1.3 \times 10^{18}\right) x^{5}+\left(2.2 \times 10^{17}\right) x^{6}-\left(3.0 \times 10^{16}\right) x^{7} \\
& +\left(3.9 \times 10^{15}\right) x^{8}-\left(4.2 \times 10^{14}\right) x^{9}+\left(4.4 \times 10^{13}\right) x^{10}-\left(3.8 \times 10^{12}\right) x^{11} \\
& +\left(3.3 \times 10^{11}\right) x^{12}-\left(2.4 \times 10^{10}\right) x^{13}+\left(1.8 \times 10^{9}\right) x^{14}-\left(1.1 \times 10^{8}\right) x^{15} \\
& +\left(7.1 \times 10^{7}\right) x^{16}-\left(3.7 \times 10^{5}\right) x^{17}+\left(1.8 \times 10^{4}\right) x^{18}-\left(6.0 \times 10^{2}\right) x^{19} \\
& \left.\left.+\left(1.5 \times 10^{1}\right) x^{20}\right)\right) \\
& y_{0}=-\left(\frac{1}{8}\right)(-4+x) x
\end{aligned}
$$




$$
\begin{aligned}
& y_{1}=\frac{1}{32768\left(-1+\mathrm{e}^{8}\right)} \mathrm{e}^{-\mathrm{x}}\left(-235940864 \mathrm{e}^{6}-235940864 \mathrm{e}^{2+2 \mathrm{x}}+\mathrm{e}^{\mathrm{x}}(-32525568\right. \\
& +31367680 \mathrm{x}-16266880 \mathrm{x}^{2}+5225216 \mathrm{x}^{3}-1355232 \mathrm{x}^{4}+262080 \mathrm{x}^{5}-45584 \mathrm{x}^{6}+6240 \mathrm{x}^{7} \\
& \left.-750 \mathrm{x}^{8}+60 \mathrm{x}^{9}-3 \mathrm{x}^{10}\right)+\mathrm{e}^{8+\mathrm{x}}\left(32525568-31367680 \mathrm{x}+16266880 \mathrm{x}^{2}-5225216 \mathrm{x}^{3}\right. \\
& \left.+1355232 \mathrm{x}^{4}-262080 \mathrm{x}^{5}+45584 \mathrm{x}^{6}-6240 \mathrm{x}^{7}+750 \mathrm{x}^{8}-60 \mathrm{x}^{9}+3 \mathrm{x}^{10}\right) \text { ) } \\
& \mathrm{y}_{2}=\left(9.1 \times 10^{-20}\right) e^{-1.0 x}( \\
& \left(-1.3 \times 10^{2}\right) e^{2}+2.0 x\left(-3.1 \times 10^{22}+\left(1.2 \times 10^{15}\right) x-\left(1.2 \times 10^{15}\right) x^{2}+\left(8.2 \times 10^{14}\right) x^{3}\right. \\
& -\left(4.1 \times 10^{14}\right) x^{4}+\left(1.7 \times 10^{14}\right) x^{5}-\left(5.9 \times 10^{13}\right) x^{6}+\left(1.7 \times 10^{13}\right) x^{7} \\
& \left.-\left(3.5 \times 10^{12}\right) x^{8}+\left(4.8 \times 10^{11}\right) x^{9}-\left(4.0 \times 10^{10}\right) x^{10}+\left(1.5 \times 10^{9}\right) x^{11}\right) \\
& +\left(1.3 \times 10^{2}\right) e^{2.0(5.0+x)}\left(-3.1 \times 10^{22}+\left(1.2 \times 10^{15}\right) x-\left(1.2 \times 10^{15}\right) x^{2}+\left(8.2 \times 10^{14}\right) x^{3}\right. \\
& -\left(4.1 \times 10^{14}\right) x^{4}+\left(1.7 \times 10^{14}\right) x^{5}-\left(5.9 \times 10^{13}\right) x^{6}+\left(1.7 \times 10^{13}\right) x^{7} \\
& \left.-\left(3.5 \times 10^{12}\right) x^{8}+\left(4.8 \times 10^{11}\right) x^{9}-\left(4.0 \times 10^{10}\right) x^{10}+\left(1.5 \times 10^{9}\right) x^{11}\right) \\
& -\left(1.5 \times 10^{8}\right)\left(3.1 \times 10^{22}+\left(6.8 \times 10^{12}\right) x+\left(6.8 \times 10^{12}\right) x^{2}+\left(4.6 \times 10^{12}\right) x^{3}+(2.3\right. \\
& \left.\times 10^{12}\right) x^{4}-\left(4.3 \times 10^{12}\right) x^{5}+\left(1.2 \times 10^{12}\right) x^{6}+\left(6.5 \times 10^{11}\right) x^{7}-(5.4 \\
& \left.\left.\times 10^{11}\right) x^{8}+\left(1.6 \times 10^{11}\right) x^{9}-\left(2.4 \times 10^{10}\right) x^{10}+\left(1.5 \times 10^{9}\right) x^{11}\right) \\
& +\left(3.5 \times 10^{3}\right) e^{x}\left(1.6 \times 10^{20}-\left(1.5 \times 10^{20}\right) x+\left(7.9 \times 10^{19}\right) x^{2}-\left(2.6 \times 10^{19}\right) x^{3}+(6.6\right. \\
& \left.\times 10^{18}\right) x^{4}-\left(1.3 \times 10^{18}\right) x^{5}+\left(2.2 \times 10^{17}\right) x^{6}-\left(3.0 \times 10^{16}\right) x^{7}+(3.9 \\
& \left.\times 10^{15}\right) x^{8}-\left(4.2 \times 10^{14}\right) x^{9}+\left(4.4 \times 10^{13}\right) x^{10}-\left(3.8 \times 10^{12}\right) x^{11}+(3.3 \\
& \left.\times 10^{11}\right) x^{12}-\left(2.4 \times 10^{10}\right) x^{13}+\left(1.8 \times 10^{9}\right) x^{14}-\left(1.1 \times 10^{8}\right) x^{15}+(7.1 \\
& \left.\times 10^{6}\right) x^{16}-\left(3.7 \times 10^{5}\right) x^{17}+\left(1.8 \times 10^{4}\right) x^{18}-\left(6.0 \times 10^{2}\right) x^{19}+(1.5 \\
& \left.\left.\times 10^{1}\right) x^{20}\right) \\
& -\left(6.9 \times 10^{3}\right) e^{8+x}\left(1.6 \times 10^{20}-\left(1.5 \times 10^{20}\right) x+\left(7.9 \times 10^{19}\right) x^{2}-\left(2.6 \times 10^{19}\right) x^{3}\right. \\
& +\left(6.6 \times 10^{18}\right) x^{4}-\left(1.3 \times 10^{18}\right) x^{5}+\left(2.2 \times 10^{17}\right) x^{6}-\left(3.0 \times 10^{16}\right) x^{7} \\
& +\left(3.9 \times 10^{15}\right) x^{8}-\left(4.2 \times 10^{14}\right) x^{9}+\left(4.4 \times 10^{13}\right) x^{10}-\left(3.8 \times 10^{12}\right) x^{11} \\
& +\left(3.3 \times 10^{11}\right) x^{12}-\left(2.4 \times 10^{10}\right) x^{13}+\left(1.8 \times 10^{9}\right) x^{14}-\left(1.1 \times 10^{8}\right) x^{15} \\
& +\left(7.1 \times 10^{6}\right) x^{16}-\left(3.7 \times 10^{5}\right) x^{17}+\left(1.8 \times 10^{4}\right) x^{18}-\left(6.0 \times 10^{2}\right) x^{19} \\
& \left.+\left(1.5 \times 10^{1}\right) x^{20}\right) \\
& +\left(3.5 \times 10^{3}\right) e^{16+x}\left(1.6 \times 10^{20}-\left(1.5 \times 10^{20}\right) x+\left(7.9 \times 10^{19}\right) x^{2}-\left(2.6 \times 10^{19}\right) x^{3}\right. \\
& +\left(6.6 \times 10^{18}\right) x^{4}-\left(1.3 \times 10^{18}\right) x^{5}+\left(2.2 \times 10^{17}\right) x^{6}-\left(3.0 \times 10^{16}\right) x^{7} \\
& +\left(3.9 \times 10^{15}\right) x^{8}-\left(4.2 \times 10^{14}\right) x^{9}+\left(4.4 \times 10^{13}\right) x^{10}-\left(3.8 \times 10^{12}\right) x^{11} \\
& +\left(3.3 \times 10^{11}\right) x^{12}-\left(2.4 \times 10^{10}\right) x^{13}+\left(1.8 \times 10^{9}\right) x^{14}-\left(1.1 \times 10^{8}\right) x^{15} \\
& +\left(7.1 \times 10^{7}\right) x^{16}-\left(3.7 \times 10^{5}\right) x^{17}+\left(1.8 \times 10^{4}\right) x^{18}-\left(6.0 \times 10^{2}\right) x^{19} \\
& \left.\left.+\left(1.5 \times 10^{1}\right) x^{20}\right)\right)
\end{aligned}
$$




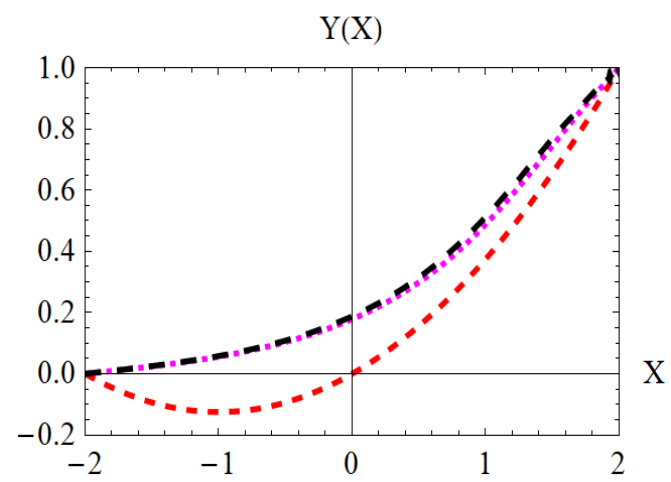

(i)

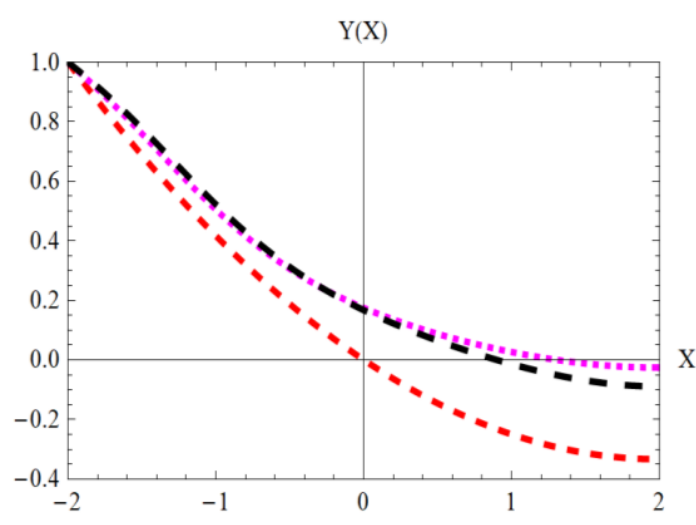

(iii)

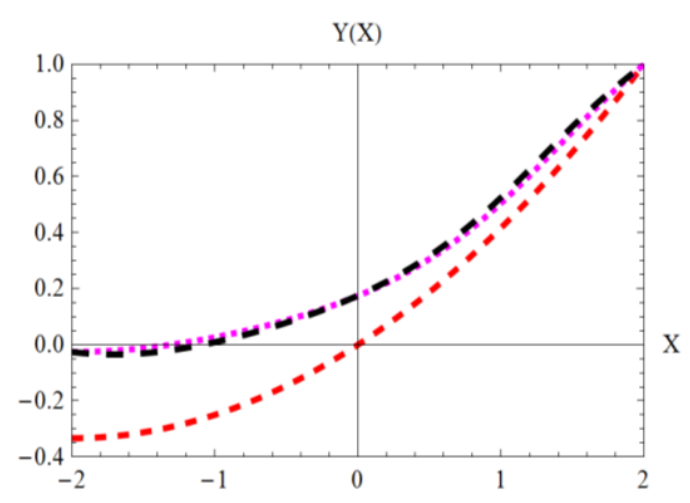

(ii)

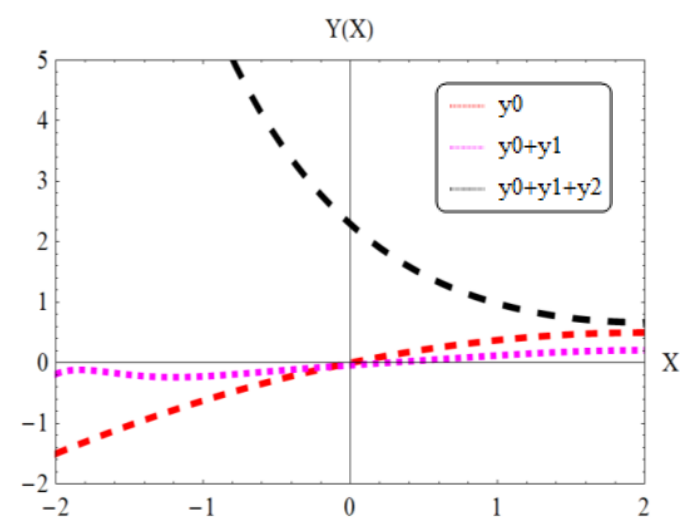

(iv)

Fig. 3: Approximate HPM solutions for boundary conditions

In Figs. 3(i, ii, iii), the dashed curve is the initial approximation $y=y_{0}$, the shaded curve is the approximation of $y=y_{0}+y_{1}$ which is the two term solution by HPM and finally the thin curve is the approximation of $y=y_{0}+y_{1}+y_{2}$ which is the three term solution by HPM. It is observed that the two and three term solutions by HPM almost coincide and may vary with the initial approximation. It can be concluded that three terms solution is the best approximate solution of Liénard differential equation with the given boundary conditions.Figure 3(iv) presents the 1term, 2-terms and 3-terms solutions of the Liénard differential equation with $u^{\prime}(a)=\alpha, u^{\prime}(b)=$ $\beta$. It is seen that three term solution differs a lot with the initial and two terms solutions. It is difficult to make any conclusion about the accuracy of the approximate solution. In the present case, 3-terms HPM solution forms a complex function which can't be used to calculate the 4-terms HPM solution.

Above findings reveal that different types of boundary condition can be used to solve the Liénard differential equation by HPM. Two terms solution and three terms solution almost coincide. More accurate solutions can be obtained by increasing the number of terms. 
Table3: Root Mean Square Error (RMSE) \& Mean Absolute Error (MAE) for the approximate solutions (Compared with HPM3)

\begin{tabular}{ccccc}
\hline BCs & RMSE for HPM1 & RMSE for HPM2 & MAE for HPM1 & MAE for HPM2 \\
\hline I & $5.29 \mathrm{E}-02$ & $8.33 \mathrm{E}-03$ & $4.63 \mathrm{E}-02$ & $6.49 \mathrm{E}-03$ \\
\hline II & $1.98 \mathrm{E}-01$ & $1.39 \mathrm{E}-02$ & $1.78 \mathrm{E}-01$ & $1.19 \mathrm{E}-02$ \\
\hline III & $1.77 \mathrm{E}-01$ & $3.25 \mathrm{E}-02$ & $1.62 \mathrm{E}-01$ & $2.54 \mathrm{E}-02$ \\
\hline IV & $6.77 \mathrm{E}+0$ & $6.30 \mathrm{E}+0$ & $4.54 \mathrm{E}+0$ & $4.38 \mathrm{E}+0$ \\
\hline
\end{tabular}

Table 3 shows that the RMSE\& MAE for the approximate solution of HPM 2 terms solutions are less than HPM 1 term solutions compared with HPM 3 terms solutions as the base solutions. As it is known with the base of Taylor's series taking higher order terms always gives better approximations which can be found is all above cases.

\section{Conclusion}

In this research, HPM is applied for the solution of the Liénard equation with different initial and boundary conditions. One, two and three parameters HP solutions are developed and presented graphically. It is found that higher parameter shows good approximations of the analytical solution. It may conclude that HPM is a very effective technique to find the analytical solutions for highly non-linear ordinary differential equation with ICs/BCs.

\section{REFFERENCES}

[1] M. Matinfar, S.R. Bahar, \& M. Ghasemi, Solving Liénard equation by differential transform method. World Journal of Modeling and Simulation 8(2) (2012) 142-146.

[2] Z. Feng, On explicit exact solutions for the Liénard equation and its applications. Physics Letters A 239 (2002) 50-56

[3] D. Kong, Explicit exact solutions for the Liénard equation and its applications. Physics Letters A 196 (1995) 301-306.

[4] J.H. He, Homotopy perturbation technique. Computer Methods in Applied Mechanics and Engineering, 178 (1999) 257-262.

[5] J.H. He, An elementary introduction to the Homotopy perturbation method. Computers and Mathematics with Applications, 57 (2009) 410-412.

[6] A. Rafiq, M. Ahmed, \& S. Hussain, A general approach to specific second order ordinary differential equations using Homotopy perturbation method. Physics Letters A 372 (2008) 4973-4976.

[7] M.S.H. Chowdhury, \& I. Hashim, Solutions of Emden-Fowler equations by Homotopy perturbation method. Nonlinear Analysis: Real World Applications 10 (2009) 104-115.

[8] A. Yıldırım, \& T. Özis, Solutions of singular IVPs of Lane-Emden type by Homotopy perturbation method. Physics Letters A 369 (2007) 70-76.

[9] A. Saadatmandia, M. Dehghan, \& A. Eftekharia, Application of He's Homotopy perturbation method for non-linear system of second-order boundary value problems. Nonlinear Analysis: Real World Applications 10 (2009) 1912-1922.

[10] G. Villari, Periodic solutions of Liénard's equation. Journal of Mathematical Analysis and Applications 86 (1982) 379-386. 
[11] G. Villari, On the existence of periodic solutions for Liénard's equation. Nonlinear Analysis, Theory, Methods \& Applications 7(1) (1983) 71-78.

[12] G. Villari, On the Qualitative behavior of solutions of Liénard's Equation. Journal of Differential Equations 67 (1987) 269-277.

[13] Z. Feng, Exact solutions to the Liénard's equation and its applications. Chaos, Solutions and Fractals 21 (2004) 343-348.

[14] M. Matinfar, H. Hosseinzadeh, \& M. Ghanbari, A numerical implementation of the variational iteration method for the Liénard's equation. World Journal of Modeling and Simulation 4(3) (2008) 205-210. 